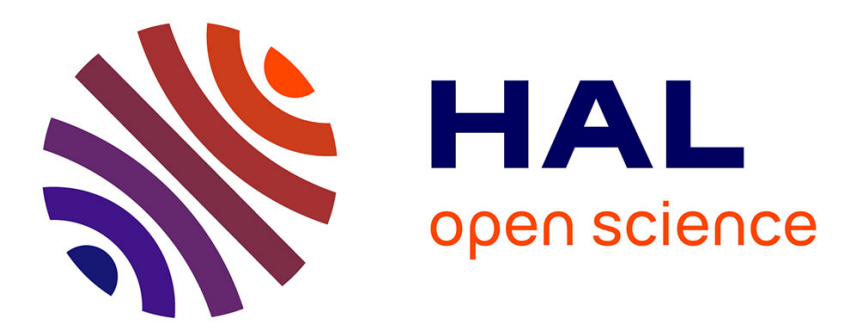

\title{
Coexisting periodic regimes in semiconductor lasers with optical feedback
}

Michael J Wishon, Anton V Kovalev, Daeyoung Choi, David S Citrin, Evgeny

A Viktorov, A. Locquet

\section{- To cite this version:}

Michael J Wishon, Anton V Kovalev, Daeyoung Choi, David S Citrin, Evgeny A Viktorov, et al.. Coexisting periodic regimes in semiconductor lasers with optical feedback. Physics and Simulation of Optoelectronic Devices XXVII, Feb 2019, San Francisco, France. pp.23, 10.1117/12.2510499 . hal-03084729

\section{HAL Id: hal-03084729 \\ https://hal.science/hal-03084729}

Submitted on 21 Dec 2020

HAL is a multi-disciplinary open access archive for the deposit and dissemination of scientific research documents, whether they are published or not. The documents may come from teaching and research institutions in France or abroad, or from public or private research centers.
L'archive ouverte pluridisciplinaire HAL, est destinée au dépôt et à la diffusion de documents scientifiques de niveau recherche, publiés ou non, émanant des établissements d'enseignement et de recherche français ou étrangers, des laboratoires publics ou privés. 


\title{
Coexisting periodic regimes in semiconductor lasers with optical feedback
}

\author{
Michael J. Wishon ${ }^{\mathrm{a}, \mathrm{b}}$, Anton V. Kovaleve, ${ }^{\mathrm{c},}$, Daeyoung Choi ${ }^{\mathrm{a}, \mathrm{b}}$, David S. Citrin ${ }^{\mathrm{a}, \mathrm{b}}$, \\ Evgeny A. Viktorov ${ }^{\mathrm{c}}$, Alexandre Locquet ${ }^{\mathrm{a}, \mathrm{b}}$ \\ ${ }^{a}$ Georgia Tech-CNRS UMI 2958, Georgia Tech Lorraine, 2 Rue Marconi, 57070 Metz, France; \\ ${ }^{\mathrm{b}}$ School of Electrical and Computer Engineering, Georgia Institute of Technology, Atlanta, Georgia \\ 30332-0250, USA; \\ 'ITMO University, Birzhevaya Liniya 14, 197341 Saint Petersburg, Russia
}

\begin{abstract}
We explore experimentally and theoretically the dynamics of a DFB quantum well laser subject to external optical feedback from a mirror. With increasing feedback, the system exhibits the following dynamical scenario: an extremely small limit cycle appears first and is followed by a quasi-periodic regime, and then by three subsequent limit cycles with different repetition rates. This sequence of limit cycles can be associated with the change of phase of the reflected field which reveals translational symmetry and the fact of periodic solutions coexistence which we confirm numerically. The results can be useful for applications in reservoir computing with phase space of coexisting limit cycles acting as a nonlinear reservoir as well as for other applications.
\end{abstract}

Keywords: laser dynamics, semiconductor lasers, optical feedback, bifurcations, periodic regimes, multistability

\section{INTRODUCTION}

Semiconductor lasers subject to optical feedback have been the focus of research for the last 50 years, but only recently the route to chaos has been correctly characterized. Due to the importance of these systems not just for nonlineardynamical studies, but for a number of emerging applications, understanding the fundamental aspects of these systems remains a priority. In this contribution, we explore experimentally and theoretically the dynamics of a DFB quantum well (QW) laser subject to external optical feedback from a mirror. The laser is characterized with high damping of side modes in the absence of feedback. The feedback loop includes a polarizer and a rotating waveplate to control the feedback rate.

It was shown previously ${ }^{1,2}$ that as the pump current increases such setup demonstrates the discretization of the relaxation oscillation frequencies and the frequencies of the self-sustained oscillations appearing at the first Hopf bifurcations when the optical feedback rate is varied. Here we explore the dynamics occurring after these bifurcations.

\section{EXPERIMENTAL SETUP AND RESULTS}

The experimental setup scheme is pictured in Figure 1. The laser is a single longitudinal-mode multi-quantum well InGaAsP DFB laser emitting at $1.55 \mathrm{um}$ characterized with high $40 \mathrm{~dB}$ damping of side modes in the absence of feedback. The feedback loop includes a polarizer and a rotating waveplate to control the feedback rate. The laser intensity signal is captured by a fast photodiode and analyzed by means of an oscilloscope and an RF spectrum analyzer. 


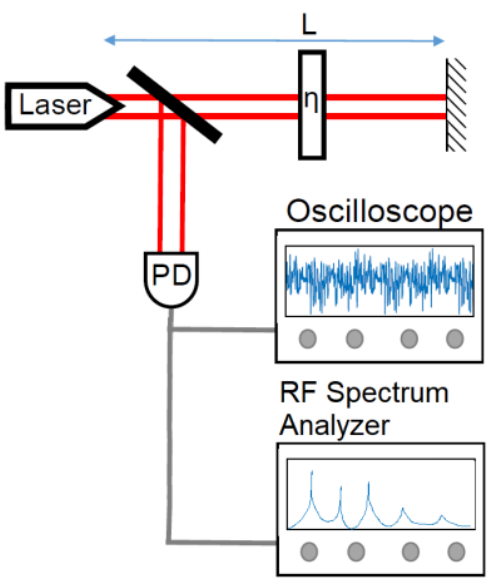

Figure 1. Experimental setup: PD is a photodiode, $\eta$ is the quarter-wave plate, and $L$ is the external cavity length.

The laser is operated at the pump current twice the threshold current which was $38 \mathrm{~mA}$. When the quarter-wave plate is rotated, the feedback strength increases and a small limit cycle appears at one of the relaxation oscillation frequencies which form a discrete set determined by the relaxation frequency of the free running laser and the external cavity repetition rate r.2. $^{1,2}$

With the feedback strength increase, the system exhibits the following dynamical scenario: a small limit cycle is followed by a quasi-periodic regime, and three subsequent limit cycles are observed next. These cycles are born from different external cavity modes (ECMs) and with different repetition rates which form a discrete frequency set. Experimental bifurcation diagrams for various pump levels and the feedback strength increase are pictured in Figure 2.

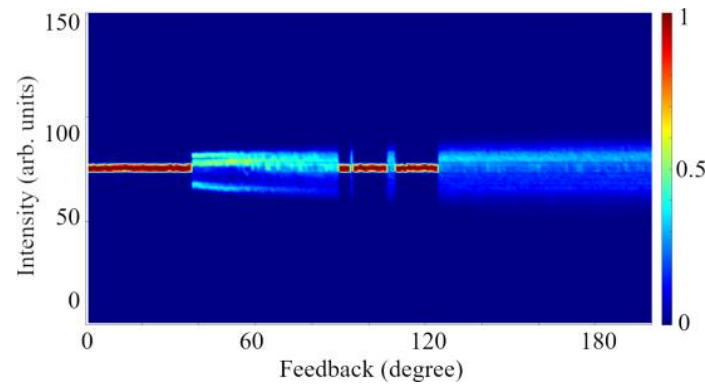

(a)

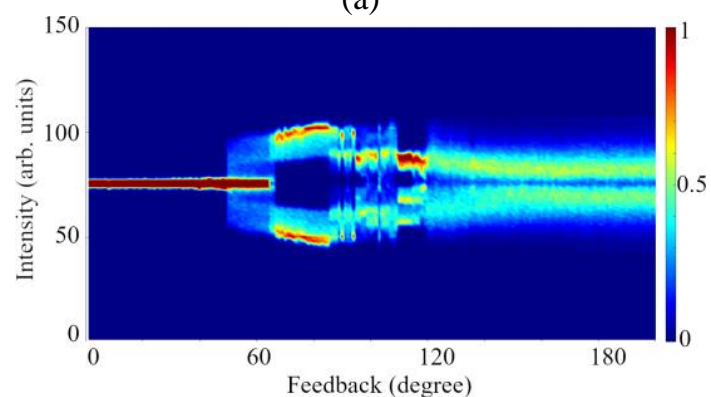

(c)

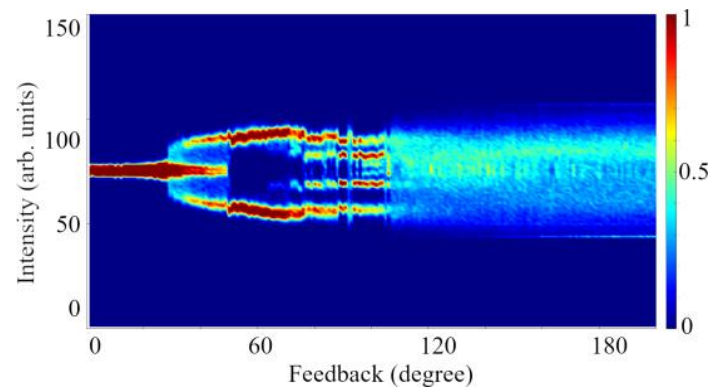

(b)

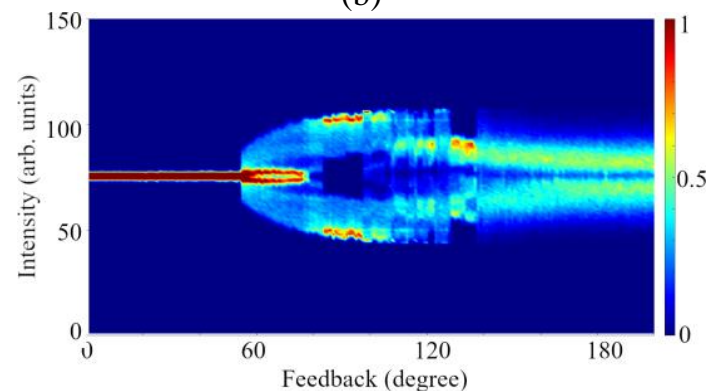

(d)

Figure 2. Experimental bifurcation diagrams showing the maxima of the laser intensity vs the feedback strength determined as the quarter-wave plate angle with respect to the polarization direction defined by the polarizer: (a) pump at 1.3 threshold values, the first Hopf Frequency is $5 \mathrm{GHz}$; (b) $9 \mathrm{GHz}$; (c) $10 \mathrm{GHz}$; (d) $11 \mathrm{GHz}$. 


\section{NUMERICAL MODELING}

The delay differential equation model of a QW laser with optical feedback reads ${ }^{3,4}$ :

$$
\begin{gathered}
\gamma^{-1} \frac{d E(t)}{d t}+E(t)=\sqrt{\kappa} \exp ((1-i \alpha) G(t-\tau) / 2-i \varphi) E(t-\tau)+ \\
\varepsilon \exp ((1-i \alpha) G(t-\tau-T) / 2-i \psi) E(t-\tau-T) \\
\frac{d G(t)}{d t}=g_{0}-\gamma_{g} G(t)-\left(e^{G(t)}-1\right)|E(t)|^{2},
\end{gathered}
$$

where $E(t)$ is the complex field amplitude; $t$ is dimensionless time normalized by the short (free running) cavity round trip time $\tau ; T$ is delay corresponding to the long (external) cavity roundtrip time; $\gamma$ is the dimensionless narrow bandwidth of the laser cavity; $\kappa<1$ is the total non-resonant round-trip intensity attenuation in the short cavity; $\varepsilon$ is the feedback coefficient; the phases $\varphi$ and $\psi$ are the parameters determined by the detuning of the frequency of the gain maximum from the optical frequency of the closest cavity mode; $G(t)$ is the normalized gain; $\alpha$ is the linewidth enhancement factor; $g_{0}$ is the dimensionless pump parameter; $\gamma_{g}$ accounts for the dimensionless carrier recombination rate.

The parameters for the analysis are: $\gamma=4 ; \tau=1 ; \mathrm{T}=120 ; \kappa=0.2, \alpha=0 ; \varphi$ and $\psi=0$. The threshold value for these parameters is $g_{0, t h r}=0.1748$, and the numerical steady state bifurcation diagram in the $\left(\varepsilon, g_{0}\right)$ plane is given in Figure 3 .

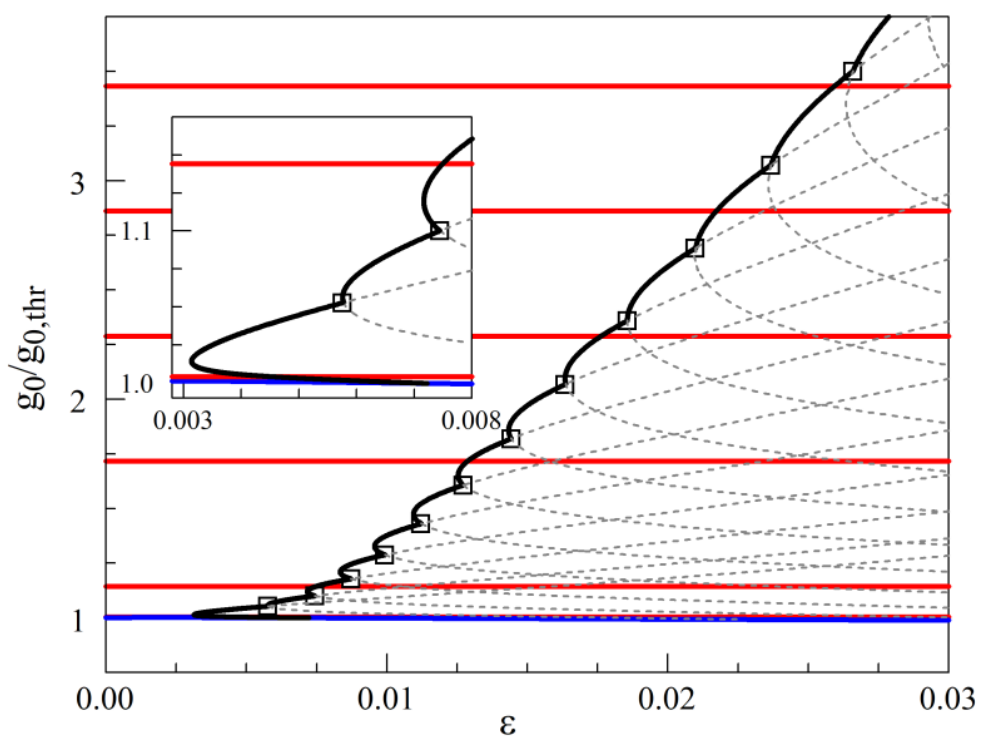

Figure 3. Numerically obtained bifurcation diagram in $\left(\varepsilon, g_{0}\right)$ parameter space at different scales. Bold black lines are for the first Hopf bifurcations of non-zero steady state solutions. Thin grey dashed lines are for continuation of the Hopf bifurcations. A blue line is the Hopf bifurcation of the laser off state. Squares are Hopf-Hopf bifurcation points. Red lines are the $g_{0}$ values selected for the bifurcation analysis. The other parameters are given in the text.

The bifurcation diagrams for selected $g_{0}$ values obtained by direct numerical integration while slowly sweeping the feedback parameter $\varepsilon$ are shown in Figure 4. These diagrams demonstrate the striking resemblance to the experimental bifurcation diagrams (see Figure 2), however there are some differences, which we address to change of the phase of the optical field, occurring with rotation of the waveplate when the feedback rate is varied. In the model, this change corresponds to the variation of the parameter $\psi$. 


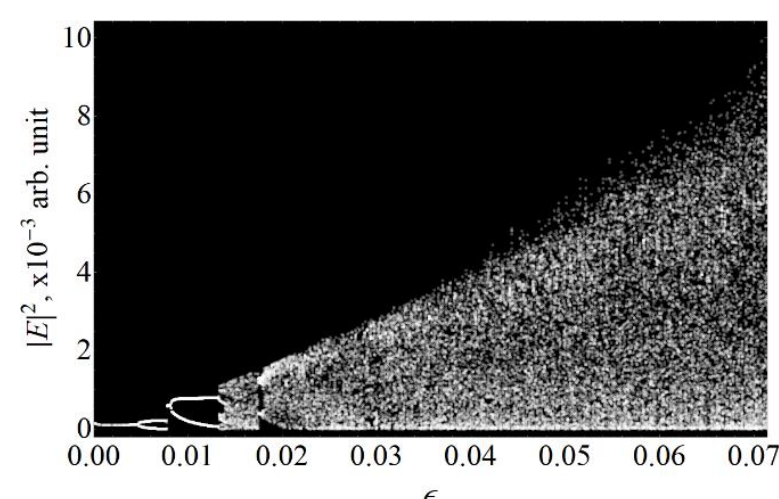

(a)

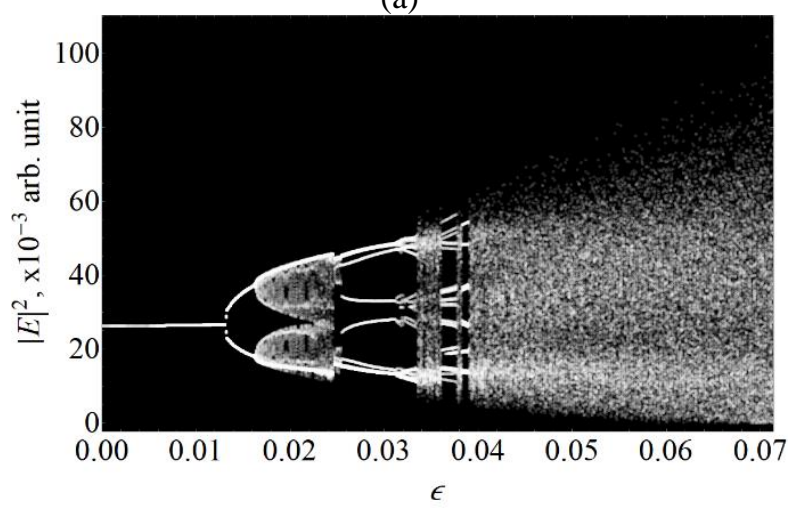

(c)

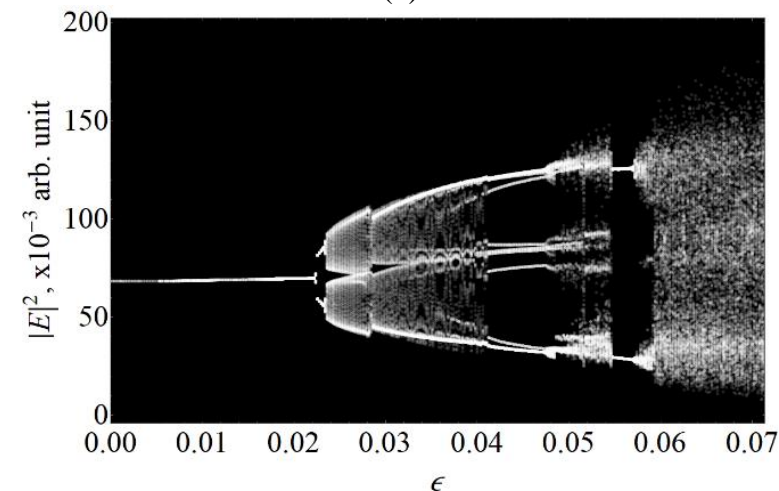

(e)

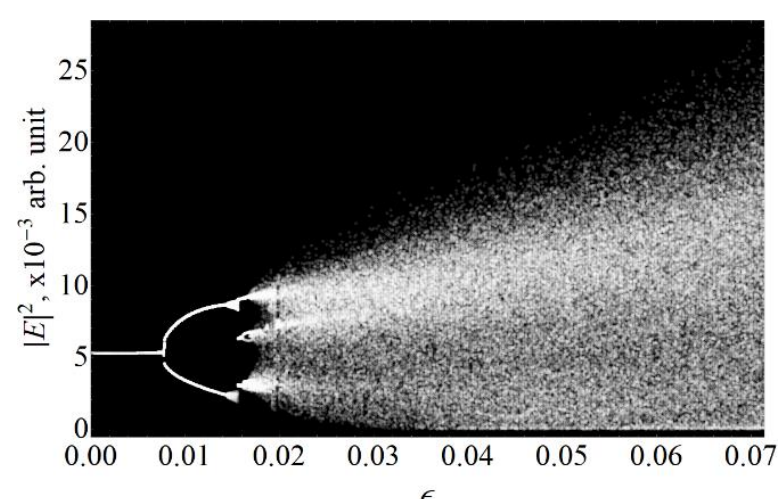

(b)

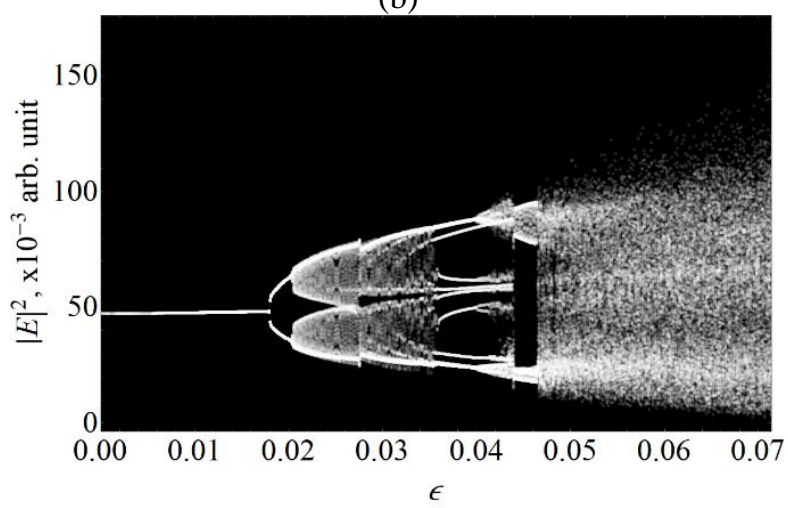

(d)

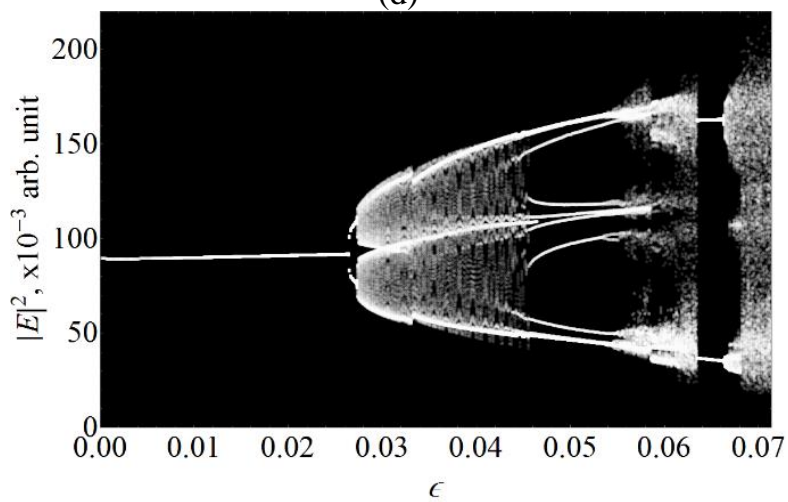

(f)

Figure 4. Numerical bifurcation diagrams for forward sweep over the parameter $\varepsilon$ for various pump parameter values corresponding to red lines in Figure 3: (a) $g_{0}=1.004 g_{0, \text { thr }}$; (b) $g_{0}=1.14 g_{0, \text { thr }}$; (c) $g_{0}=1.72 g_{0, \text { thr }}$; (d) $g_{0}=2.29 g_{0, \text { thr }}$; (e) $g_{0}=$

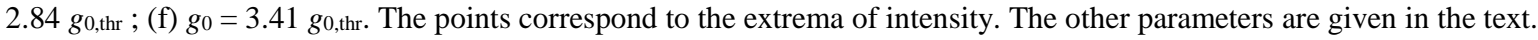

The change of the feedback strength parameter $\varepsilon$ sweep direction reveals coexistence and bistability of dynamical regimes of the system as demonstrated in Figure 5. 


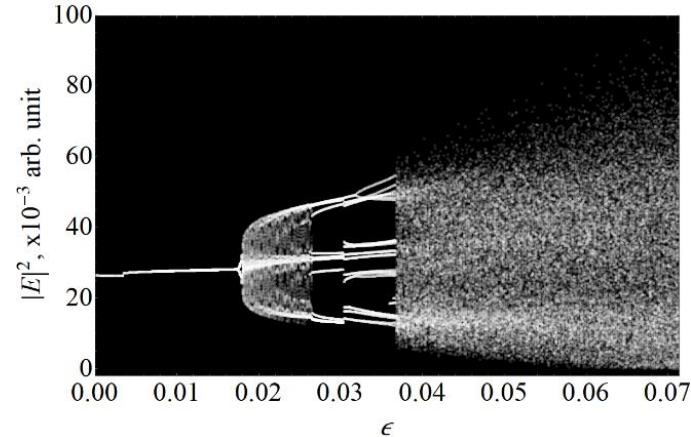

(a)

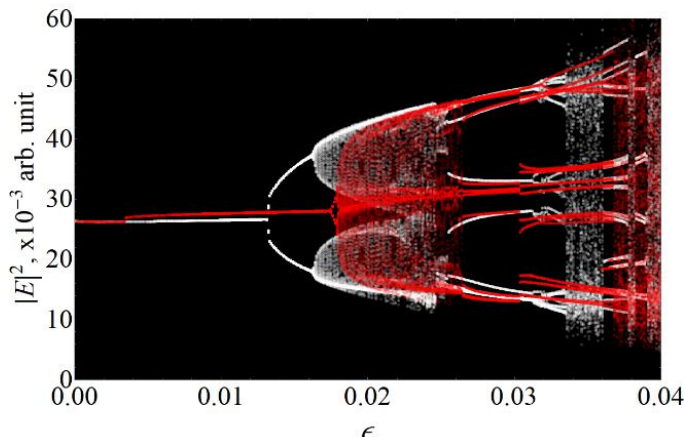

(b)

Figure 5. (a) Numerically obtained bifurcation diagram for slow sweeping backward across the parameter $\varepsilon$ for $g_{0}=1.72$

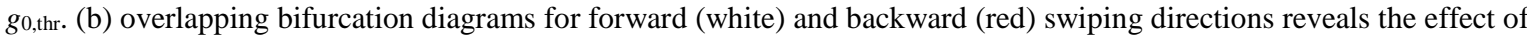
bistability. The points correspond to the extrema of intensity. The other parameters are given in the text.

Variation of the feedback phase parameter $\psi$, reveals coexistence of the multiple dynamics states, and a possibility of switching between them. This multistability indicates 2pi translational symmetry of the Eqs (1)-(2) explains the that the experimental periodic solutions actually coexist (see Figure 6(a)). Results of simultaneous variation of the feedback strength and the feedback phase are shown in Figure 6(b,c). We assume a linear relationship between the phase and the feedback strength as indicated in the caption. The difference between the two bifurcation diagrams in Figure 6(b, c)) emphasizes an impact of the feedback phase variation on the bifurcation sequence and suggests that the variation should be taken into account in the modeling aiming to reproduce the experimental results.

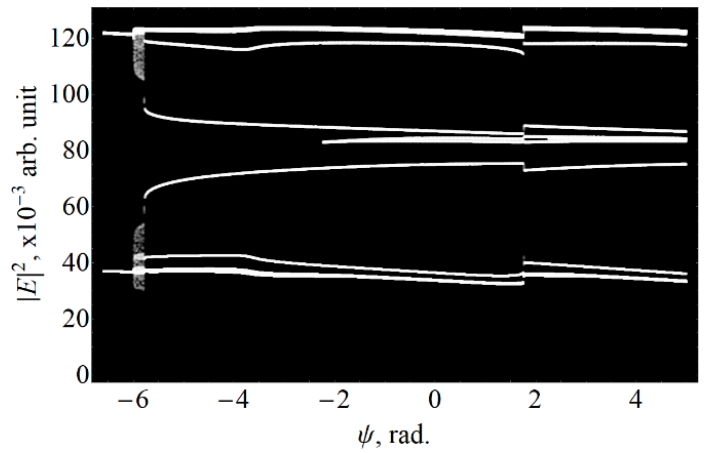

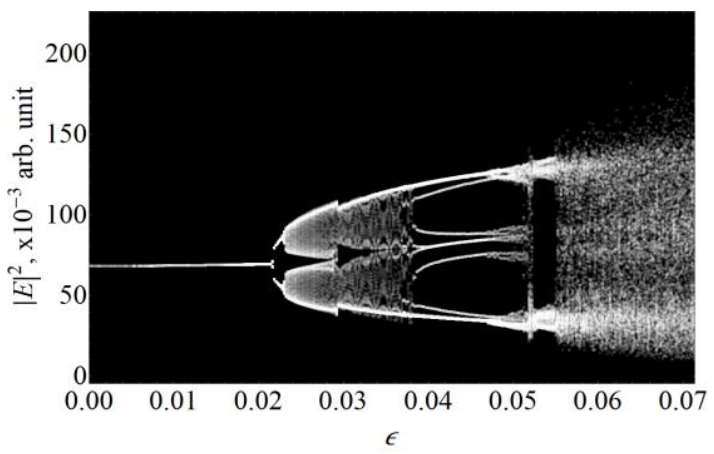

(b)

(a)

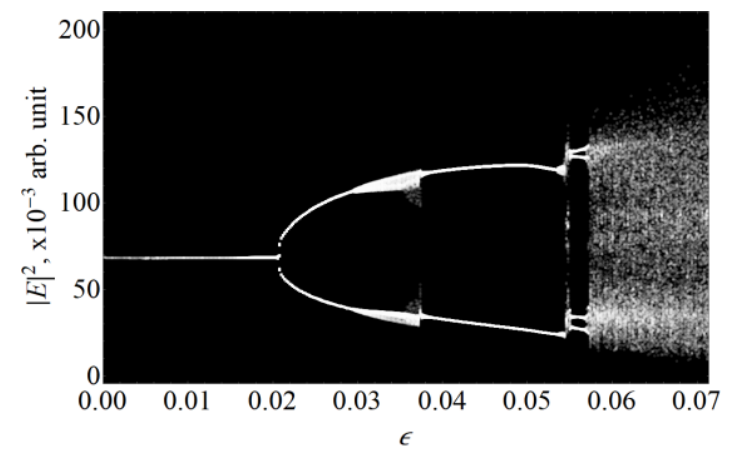

(c)

Figure 6. (a) Bifurcation diagram for slow sweeping the feedback phase parameter $\psi$ for $g_{0}=2.29 g_{0, \text { thr }}$ and $\varepsilon=0.044$. (b-c) The bifurcation diagrams for sweeping both feedback phase $\psi$ and strength $\varepsilon$ parameters for $g_{0}=2.29 g_{0, \text { thr }}$ where the relations between the sweeping steps are $\delta \psi=75 \delta \varepsilon(\mathrm{b})$, and $\delta \psi=150 \delta \varepsilon(\mathrm{c})$. 


\section{CONCLUSION}

Experimentally and numerically, we have demonstrated a bifurcational sequence indicating coexistence of multiple periodic regimes in a semiconductor laser with optical feedback. We find that coexisting limit cycles can be explained by the variation of the feedback phase which appears simultaneously with the variation of the feedback strength if rotation of the waveplate is used to control the feedback. The effect of coexistence reveals translational symmetry of the semiconductor laser system subject to optical feedback, which we have confirmed numerically. The results can be useful for applications in reservoir computing with phase space of coexisting limit cycles acting as a nonlinear reservoir as well as for other applications.

\section{REFERENCES}

[1] Kelleher, B., Wishon, M. J., Locquet, A., Goulding, D., Tykalewicz, B., Huyet, G., and Viktorov, E. A., "Delay induced high order locking eects in semiconductor lasers," Chaos: An Interdisciplinary Journal of Nonlinear Science 27(11), 114325 (2017).

[2] Kovalev, A.V., Tykalewicz, B., Goulding, D., Kelleher, B., Wishon, M.J., Locquet, A., Citrin D., and Viktorov, E.A., "Feedback-induced discretisation of the relaxation oscillation frequency in a semiconductor laser," Proc. SPIE 10682, 106821I (2018).

[3] Otto, C., Ludge, K., Vladimirov, A. G., Wolfrum, M., and Scholl, E., "Delay-induced dynamics and jitter reduction of passively mode-locked semiconductor lasers subject to optical feedback," New Journal of Physics 14(11), 113033 (2012).

[4] Vladimirov, A. G. and Turaev, D., "Model for passive mode locking in semiconductor lasers," Physical Review A 72(3), 033808 (2005). 